\title{
Cloning and characterization of the carp prolactin gene
}

\author{
Huang-Tsu Chen ${ }^{1}$, Chien-Shun Chiou ${ }^{1}$ and Wen-Chang Chang 1.2

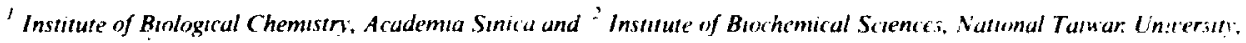 \\ Taipei (Tawan, (hina)
}

(Received 21 November 1990)

Key words: Prolactin: Genomic sequence: Palindrome; (Carp)

A carp genomic DNA clone containing the carp prolactin (Prl) gene was isolated with carp Prl cDNA as a probe. The organization of the carp $P \boldsymbol{H}$ gene was determined by restriction nuclease mapping and nucleotide sequencing. The $P \boldsymbol{r}$ gene comprises approx. 2.8 kilobasepairs (kb) of DNA including the 5 '-flanking region, five exons, four introns and the $3^{\prime}$-flanking region. Analysis of the $5^{\prime}$-flanking region reveals (1) the sequence TATATAAT at positions - 38 to -31 upstream from the cap site which was found to be a guanine resitue, and (2) the palindrome, CTCATTGCATATACAAATGAG at positions -79 to -59 . The carp $P r$ gene matches with the reported cDNA except for one difference in coding region and five in the $3^{\prime}$-flanking region, while the encoded amino acid sequences are identical. The arrangemerit of exons and introns is very similar to that seen in carp $G H$ as well as mammalian Prl, which, however, have much longer intron:s.

(A)

EMBL 3

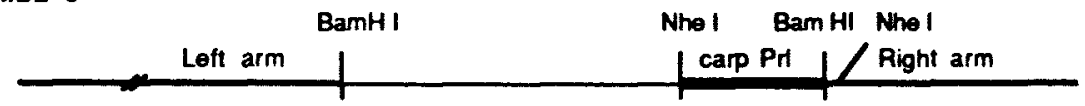

(B)

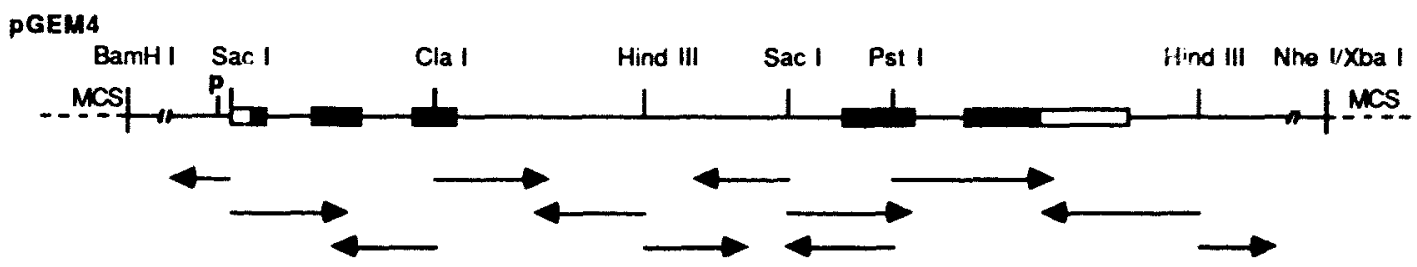

Fig. 1. The restriction map of carp Prl gene in EMBL 3 (A) and the sequencing strategy for the subcloned tragment in pOEM 4 (B). The arrows show: the direction of sequencing by the chdin-termination method. P. TATA box: MCS. multiple cloning site; solid box, coding region; open box. untranslated region: lines between the boxes, introns.

The sequence data in this paper have been submitted to the EMBL/Genbank Data Lib. ries under the accession number $\times 52881$ Abbraviations: Prl, prolactin: GH. growth hormone

Correspondence: W.C. Chang. Institute of Biolugical Chemistry. Academia Sinica. P.O. Box 23-106. Taipei. Taiwan.
Prolactin ( $\mathrm{Prl})$ is a polypeptide hormone produced and secreted by the anterior pituitary gland [1]. It is a member of a family of hormones that includes Prl, growth hormone (GH) and placental lactogen. This family is believed to have evolved from a single ancestral gene by duplication and sequence divergence [2]. 
atagctcataacattgatcagccaaccctcaatcaatgttttcattagttcaaggtttactaatacacctggagtgcaagact $+1$

cattgcatatacaaatgagaggaccaagaagtgcaggcctatataatgggaagaaaatgacagagagctcaagagag $G$ AAATCAGCTG CAAAGATTCAAGCGAGAGAATAAAGAAAACAGTTAAA ATG ACT CAA GGA TCT AGA CTA TAC TTT GCA G gtaggCactc

$$
\begin{array}{lllllllllll}
M & T & Q & G & S & R & L & Y & F & A
\end{array}
$$

ctccattcctcataacatcccetttttatttctgttcatacttattagacttcagatgactatactgttatttttgaacagtt at ttctigttgctttcag TG ACT GTT CTG ATG TGT GCG TTT GIC TCC ATC AAT GGT GTC GGT CTG AAT GAT $\begin{array}{llllllllllllllllllll}V & T & V & L & M & C & A & F & V & S & I & N & G & V & G & L & N & D\end{array}$ TTA CTG GAG CGA GCC TCT CAA CTT TCA GAC AAA CTC CAC TCT CTC AGC ACT TCT CTC ACC AAT GAC $\begin{array}{llllllllllllllllllllllll}L & L & E & R & A & S & Q & L & S & D & K & L & H & S & L & S & T & S & L & T & N & D\end{array}$ CTG gtcagtaccettgtaacgtgtttggtgttatatgcacaatcact attegcagtatgtgacttgagttttatccaadagatatt L

tattcattgattgattggttgttagatattaaccttgcttcatccacag GAT TCT CAC TTT CCT CCT GTT GGT AGG GTA ATG ATG CCT CGT CCA TCG ATG TGC CAC ACT TCC $\quad$ TCT CTT $\begin{array}{llllllllllllllllllllll} & M & M & P & R & P & S & M & C & H & T & S & S & L & Q & I & P & N & D & K & D & Q\end{array}$ GCC TTG AAA ATA CCG gtaagtaatttaactcatccatctctatcaaatgcaaaattattctcctacactctcagaaataaa A L $Q$ I $P$ atgtatgaatgctgtcactggggcagtttatttcaaaggtacactttgtatcttcaggcgctaatgtcgacactt aggtactaat atgtaggctacctt aaggtaccaatatgaaagagaccgccccocgacagcttegtaccttttttctgagagtgttttccagcttt tt at attctat att gattgattttataattaaaatattaatat atacagcatattaaaaaaatagaatagaaaaat acattact aattgttgttatactattatatttatatacaacaaataacaaaatacaaadacgtgatactatgatgcaagcttro aacatgtgaagttctcataggttttttttcattcagtctcaatccaacctatttcagcatattcacacaaaatgaaattctgt catt tactcaccattgatcaacccattagactttagttgatcctcaaaacaaaagaagatattttaatgaaccacaagggatt ct gtccctccattgaaght cat ccaccaaactttgatccat ataat cgagctgtt aatccaagt cct ctaagaaacgtcat cact cacatgatgaacagatt aatt aggtttattcacatatgaacaatactcaaacacatatagagcacattaacaataatatatga gctcagctgtacttctgcagaatcacaatgtgtgtgacctcattggttcaagtct tatggattggaacaacatgaatt acatatt tatggggtgaactt caatgtgaagtgacaaacatctcccacttgtgtcacattggtagttgcagaagaaacagaccagtagtgagtgaa gtttatctgattgtttgtgcag GAG GAT GAG CTG CTG TCT TTG GCT CGG TCT CTG CTG CTG GCG TGG TCC

GAT CCC CTC GCC CTT CTC TCC TCT GAG GCA TCC AGC CTG GCA CAT CCA GAA CGC AAC ACC ATT GAC 1672 $\begin{array}{lllllllllllllllllllllll}D & P & L & A & L & L & S & S & E & A & S & S & \text { L } & A & H & P & E & R & N & T & I & D\end{array}$ AGC AAG ACC AAA GAG CTG CAG GAC AAC ATC AAC AGC CTG GGA GCA GGT CTG GAG CAC GTC TTT CAA 1738 $\begin{array}{llllllllllllllllllllllll}S & K & T & K & E & L & Q & D & N & I & N & S & L & G & A & G & L & E & H & V & F & Q\end{array}$ AAGgtgattgtactcacagtgcat at attgtgaatgttgattggaagagggcaatatgaagttattcaaaat atgaagaaatctttt K

aagcaaggttggtttatacatcgtccetgtccgtgttttcttctclacag ATG GGC TCA TCT TCA GAC AAC CTG TCC TCT 1828 $\begin{array}{llllllllll}M & G & S & S & S & D & N & L & S & S\end{array}$ CTC CCT TTT TAC ACC AGC AGC CTT GGC CAG GAT AAN ACT TCT CGA CTT GTC AAT TTC CAT TTC CTG $\begin{array}{llllllllllllllllllllllll}L & P & F & Y & T & S & S & L & G & Q & D & K & T & S & R & L & V & N & F & H & F & L\end{array}$ CTG TCC TGT TTC CGC AGG GAC TCC CAC AAA ATT GAC AGT TTC CTC AAA GTT CTC CGC TGT CGG GCA $\begin{array}{lllllllllllllllllllllll}L & S & C & F & R & R & D & S & H & K & I & D & S & F & L & K & V & L & R & C & R & A\end{array}$ GCC AAG AAG AGA CCT GAG ATG TGT TAG AGTGGAAATGTGTGCTCTGCTTCTCTCAGTGTGGATGTTAAGTTAAAATGGC $\begin{array}{llllllll}A & K & K & R & P & E & M & C\end{array}$ AGAGCTGTGAGTGATTTGAAAATCAAATGGTAAAATATGGTCTTTATTGGTTGTTTCAAAGATATAGATATTTGATTTACTIGAATAAAT C $\$$ G

ACCTGGACCAACATCATAGATTACTGTAATCTCAAAGATAAAAGACACCAAAATAAATACATTTTAAAAAt agaaadtgaaacacagc $C$ TA

caggttatgcaaatgtctgt atgcagtgatattgtatgatgaataaatgcatcgtatattgaaatgt ttgactagagtaactaaattgt att tat taggcagt tatgttggatggttaacagttattt aacat atct tctgtaatgtgatatt aaagcttgcaccaatgtcaat aatt tet ataatgcagatt tatat tgact catggggaaaaat gtatgcatgaaaaggactgtgaaaaaaaattctctaccact $c$ ccacacaatgtgaacatacacagaataagtgtgtaggctacacacacacacacacacacacacacacaacagtt

Fig. 2. The complete nucleotide sequence of carp Prl gene. Exons are shown by capitals and introns by lower case letters. The numbering is given in the right margin and begins at the cap site $(+1)$. Encoded amino acid residues are represented by one-letter codes below the codons. The bases in mRNA [17] are shown below the genomic sequence at positions of discrepancy indicated by asterisks. The upstream palindrome. TATA box and the down stream polyadenylation signal are underlined. 
$P r l$ gene expression is under complex hormonal control. Many studies had shown that thyroid hormone, glucocorticoids, dopamine, etc. [3-7] directly regulate the levels of Prl and/or GH mRNA. Prl and GH transcription is generally regulated in opposite direction by most of the above-mentioned hormones, providing a very interesting system to study the mechanisms of hormonal control of gene expression.

In vertebrates, Prl is involved in many physiological functions, the best known being lactogenesis [8]. In teleosts, the hormone is implicated in osmoregulation, electrolyte balance and many other functions $[9,10]$. Prunet et al. [11] found that $\mathrm{Prl}$ expression in rainbow trout might be effected by changes in water salinity. However, there is still little information concerning the role of Prl in fish adaptation to new osmotic environments $[12,13]$. These features make $P r l$ an interesting gene to study, especially with respect to the potential control sequence that may be located in the $5^{\prime}$-flanking region of this gene $[14,16]$. Analysis of the Prl gene could provide some insight into the structures which are involved in the gene expression. Furthermore, tiis may

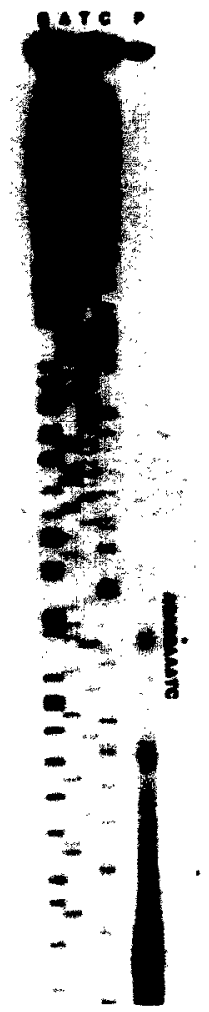

Fig. 3. Determination of the transcription start site. Lanes G. A. T and $C$ indicate the size markers from dideoxy sequencing reaction products of vector mpl8 as template with a suitable primer. Lane $P$ is the primer-extension products generated using synthetic oligonucleotide primer and mRNA. The asterisk indicates the guanine residue at transcription start site. contribute to an understanding of mechanisms which facilitate the differential expression and regulation of $\mathrm{Prl}$ gene.

As an integral part of our studies on the structure. function and molecular biology of fish pituitary hormones [17-19], the carp Prl gene was cloned, sequenced and characterized. These data will be essential for further studies on the regulation and functions of $\mathrm{Prl}$ gene in fish.

Eggs collected from common carp (Cyprinus carpio) were immediately frozen in liquid nitrogen and stored at $-70^{\circ} \mathrm{C}$. Genomic DNA was prepared from the carp eggs and partially digested with BamHI. Fragments of $10-20 \mathrm{~kb}$ were recovered from $0.5 \%$ agarose gel and ligated with $\lambda$ phage EMBL3 arms. In vitro package and transfection were carried out according to the protocols of the commercial supplier (Promega).

The carp genomic library was screened with ${ }^{32} \mathbf{p}$. labeled carp Frl cDNA [17] as probe. The positive clones were digested with suitable restriction enzymes and subcloned in plasmid pGEM 4. Recombinant phage and plasmid were purified by the standard procedures [20]. DNA sequence was determined by chain-termination method using denatured piasmid templates [21].

The transcription start site was determined by primer extension using carp pituitary mRNA as template. The mRNA was prepared as previously described [19] and annealed to a synthetic primer 5'-GTCTAGATCCTTGAGTCAT-3' which is complementary to the first six codons of exon 1 in the genomic sequence (Fig. 2). The primar extension was carried out with the mouse Moloney leukemia virus reverse transcriptase. The ${ }^{32} \mathrm{P}$-labeled reaction products were analyzed by electrophoresis in $10 \%$ polyacrylamide sequencing gel.

From a library of $2 \cdot 10^{5}$ plaques. one clone was found to hybridize strongly with the radiolabeled cDNA probe. Restriction enzyme mapping and hybridization with the cDNA probe revealed that the positive clone contained a $\mathrm{Prl}$ gene in an insert of about $15 \mathrm{~kb}$ (Fig. 1). The exon-containing fragments were identified by further digestion and hybridization and were subcloned in pGEM 4 vector and sequenced (Fig. 1). The complete nucleotide sequence was shown in Fig. 2 together with the encoded amino acid sequence of carp Prl. Comparison of the genomic sequence with that of the cDNA reveals identical amino acid sequence of the gene product and only one base-change in the coding region and 5 more in the $3^{\prime}$-untranslated region of the last exon (Fig. 2). Certainly this $2.7 \mathrm{~kb}$ DNA fragment contains the full-length Prl gene which would give rise to the cDNA previously cloned [17]. A TATA box was found in the $5^{\prime}$-flanking region from -38 to -31 (Fig. 2). Further upstream was a 21 bp palindrome CTCATTGCATATACAAATGAG from -59 to -79 . The CAAT box was not found in this region. All the intronexon boundaries followed the GT/AG rule [22]. 


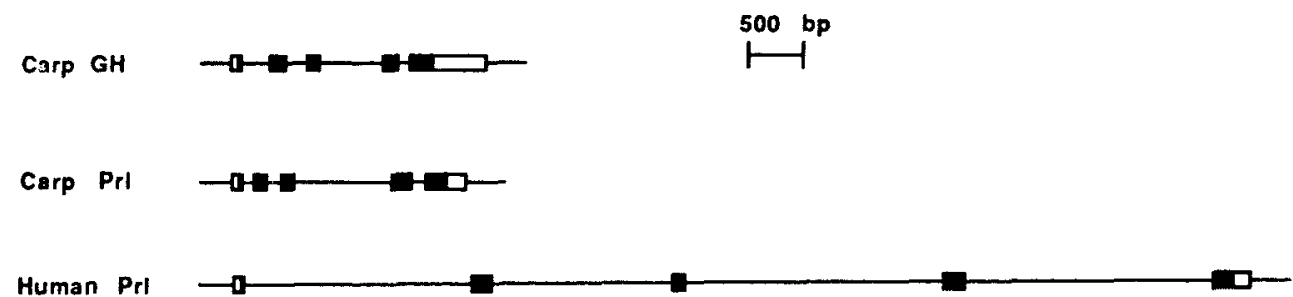

Rat PrI

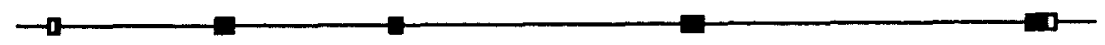

Fig. 4. Comparison of structures of the Prl genes of carp (this study). human [16] and rat [6] and of the carp GH gene [18]. Solid boxes. coding regions: open boxes, untranslated regions: lines between the boxes, introns.

The transcription start site was determined by the primer extension method (Fig. 3) and found to be a guanine residue. located 38 bp downstream from the TATA box (Fig. 2).

As shown in Fig. 4, the $P r l$ and $G H[18]$ genes in carp are very similar in terms of size, number and length of exons and introns. However, they are much shorter as compared to mammalian genes, due to the smaller introns in the carp genes as is the case for carp $\gamma$-crystallin genes (unpublished data). The carp Prl and GH also exhibit $50 \%$ homology in their coding scquence, appreciably higher than rat and human, which have around 40\% sequence homology in the $\mathrm{Prl}$ and $\mathrm{GH}$ coding regions (data not shown).

This study was supported by a grant from the $\mathrm{Na}$ tional Science Council of the Republic of China.

\section{References}

1 Niall. H.D., Hogan, M.L.. Saucr, R., Rosenblum, I.Y. and Greenwood. F.C. (1971) Proc. Natl. Acad. Sci. USA 68. 866-869.

2 Li. C.H. (1980) in Hormonal Proteins and Peptides (Li. C.H.. ed.). Vol. 8. pp. 1-36. Academic Press. New York.

3 Tashjian, A.H., Bancroft, F.C. and Levine. L. (1979) J. Cell Biol. 47. 61-70.

4 Gourc;i, D. Tougard, C. and Tixier-Vidal, A. (1982) in Frontiers in Neuroendocrinology (Ganong. W.F. and Martini, L., eds.), Vol 7. pp. 317-357, Raven Press, New York.

5 Maurer, R.A. (1981) Nature 294. 94-97.

6 Maurer. R.A.. Ermin, C.R. and Donelson. J.E. (1981) J. Biol Chem. 256. 10524-10528.
7 Murdoch, G.H.. Potter, E. Nicolaiser A.K.. Evans, R.M. and Rosenfels, M.G. (1982) Nature 300, 192-194.

8 Emmerman. J.T., Enami. J.. Pitelka. D.R. and Nandi, S. (1977) Proc. Natl. Acad. Sci. USA 74, 4466-4470.

y Clarke, W.C. and Bern. H.A. (1980) in Hormonal Proteins and Peptides. (Li. C.H.. ed.). Vol. 8. pp. 105-197, Academic Press. New York.

10 Ensor, D.M. (1978) in Comparative Endocrinology of Prolactin, pp. 15-44, Chapman and Hall, London.

11 Prunet. P.. Boeuf, G. and Houdebine. L.M. (1985) J. Exp. Zool. 235. 187-196

12 Loretz, C.A. and Bern, H.A. (1982) Neuroendocrinology 35, 292 304.

13 Hirano, T. (1986) in Comparative Endocrinology Developments and Directions (Ralph, C.L., ed.), Alan R. Liss, New York.

14 Gubbins. E.J., Maurer. R.A., Lagrimini. M., Ermin, C.R. and Donelson. J.E. (1980) J. Biol. Chem. 255, 8655-8662.

15 Camper, S.A.. Luck, D.N.. Yao. Y.. Woychi. R.P.. Goodwin. R.G., Lyons, R.H. and Rottman, F.M. (1984) DNA 3, 237-249.

16 Truong, A.T., Ducz. C., Belayew, A.. Renard. A., Pictet, R., Bell, G. and Martial. J.A. (1984) EMBO J. 3. 429-437.

17 Chao. S.C.. Pan. F.M. and Chang. W.C. (1988) Nucleic Acids Res. 16. $9350-9350$.

is Chou. C.S., Chen. H.I. and Chang. W.C. (1990) Biochim. Biophys. Acta 1087, 91-94.

19 Chao, S.C.. Pan, F.M. and Chang, W.C. (1989) Biochim. Biophys. Acta 1007. 233-236.

20 Maniatis. T.. Fritsch. E.F. and Sambrook, J. (1982) Molecular Cloning. A laboratory manual, Cold Spring Harbor Laboratory. Cold Spring Harbor. New York.

21 Hattori, M. and Sakaki. Y. (1986) Anal. Biochem. 152. 232-238.

22 Breathnach. $R$ and Chambon, P. (1981) Annu. Rev. Biochem. 50. $349-383$. 